\title{
Sarcoidosis: a rare cause of solitary pulmonary nodule
}

\begin{abstract}
Sarcoidosis is a disease of unknown cause that leads to inflammation. Sarcoidosis can affect any organ in your body. However, it's more likely to affect some organs than others. The disease usually starts in the lungs, skin, and/or lymph nodes. Lung manifestation of sarcoidosis includes hilar and mediastinal adenopathy, lung parenchymal infiltrates. However SPN as a manifestation of sarcoidosis is uncommon. We present a case of sarcoidosis presenting as SPN.
\end{abstract}

Keywords: sarcoidosis, solitary pulmonary nodule, granuloma
Volume I Issue 4 - 2014

\section{Hiren J Mehta}

Division of Pulmonary and Critical Care Medicine, University of Florida, USA

\begin{abstract}
Correspondence: Hiren J Mehta, Division of Pulmonary and Critical Care Medicine, Department of Medicine, University of Florida, Gainesville, FL, USA, Tel (352) 273-8589,
\end{abstract}

Email Hiren.Mehta@medicine.ulf.edu

Received: November 08, 2014 | Published: November 26, 2014

\section{Case report}

54year old female with past medical history of diabetes, hypertension, obstructive sleep apnea on CPAP at night and hyperlipidemia originally presented to clinic with left sided chest pains. She stated that the pains were sharp stabbing in nature and localized to left precordial area. Pain was ongoing for the last $2-3$ weeks and was slowly getting worse. No radiation of pain. She also complained of mild DOE which was stable over the last 6months. Also c/o mild cough especially in the morning and mainly dry in nature. No weight loss, night sweats, no change in appetite. She had a 35 pack year smoking history and quit smoking 3 years back. She worked as a janitor. No significant family history of malignancies or lung disorders. Her physical examination was unremarkable and her routine outpatient blood tests were within normal limits. She had a Chest $\mathrm{x}$-ray which showed a right mid zone lung nodule (Figure 1). She had a computed tomography (CT scan) of the chest (Figure 2) which showed $1.4 \times 1.7 \mathrm{~cm}$ noncalcified pulmonary nodule in the right upper lobe. She was referred to pulmonary clinic for further evaluation. Given high probability of malignancy patient underwent right thoracotomy with right upper lobe wedge resection along with partial Mediastinal lymphadenectomy. Pathology came back as localized organizing pneumonia with associated giant cell reaction and non-necrotizing granulomas with focal peribronchiolar hyaline fibrosis (Figure 3). Right paratracheal lymph node which was also excised showed anthracotic lymph node with hyalinized granulomas. Acid fast bacilli cultures and fungal culture which were taken intraoperatively were negative. Patient had negative ANA and ANCA screen and negative serum histoplasma antigen and urine cryptococcal antigen. At that point diagnosis of pulmonary sarcoidosis presenting as solitary pulmonary nodule (SPN) was made. Patient did not have any evidence of extrapulmonary disease and no other focus of pulmonary involvement was identified. Patient recovered uneventfully and no further treatment was initiated.

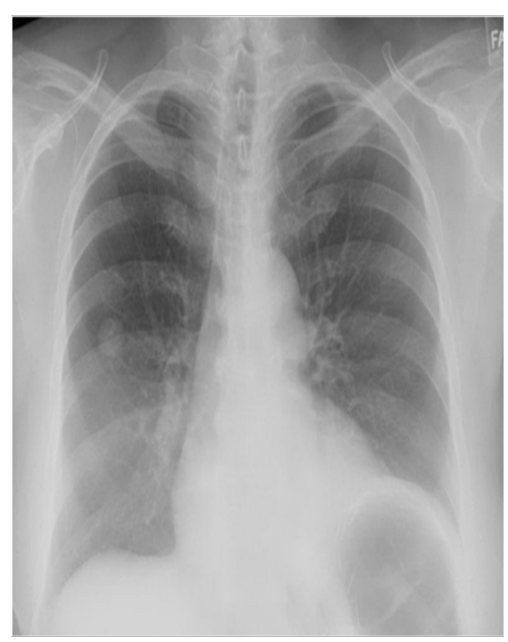

Figure I Shows right mid zone lung nodule.

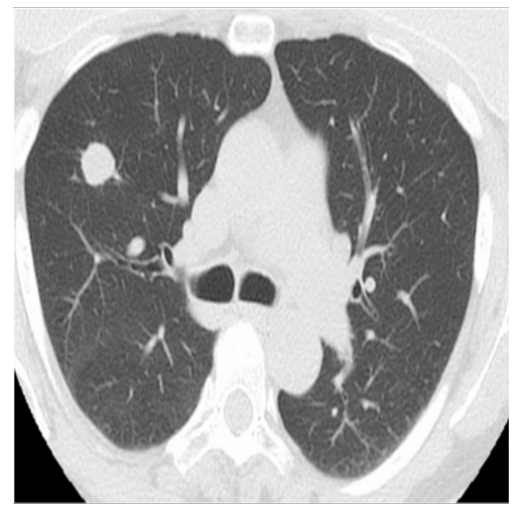

Figure 2 Shows right upper lobe lung nodule. 


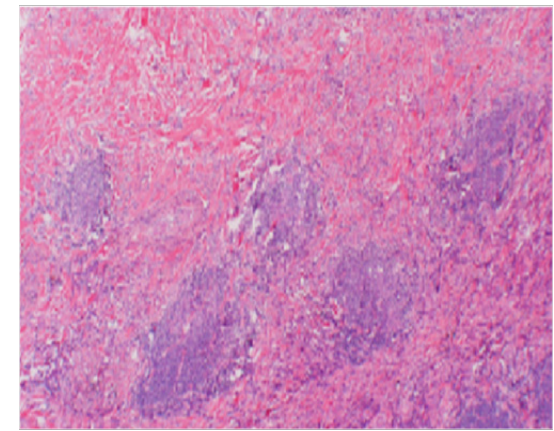

Figure 3 Non caseating granulomas suggestive of Sarcoidosis.

\section{Discussion}

Although majority of lung nodules are benign,a SPN in smokers which is $>1 \mathrm{~cm}$ in diameter is an ominous finding mandating tissue diagnosis. SPNs have an extensive differential diagnosis and the management is frequently challenging. Sarcoidosis rarely presents as isolated, discrete lung opacity. A thorough review reveals that so far there have been around 5 cases of sarcoidosis reported in literature presenting as SPN. ${ }^{1,2}$ Some other reported had bilateral hilaradenopathy along with SPN as a presenting feature. ${ }^{3}$ Majority of the cases were asymptomatic and SPN was picked up as an incidental finding. ${ }^{4,5}$ In the Veterans Administration Cooperative Study, of the total of 1134 surgically resected SPN's only 1 was found to be sarcoidosis. ${ }^{6}$

SPN is thus considered to be one of the atypical manifestations of pulmonary sarcoidosis. Other atypical manifestations of pulmonary sarcoidosis include unilateral or asymmetric adenopathy, mediastinal without hilar adenopathy, lung cavitation, pleural effusions and lower lobe predominant interstitial lung disease. ${ }^{7}$

Although an SPN occasionally cannot be preoperatively diagnosed with confidence, awareness of this presentation of sarcoidosis may favor the selection of a limited surgical procedure.

\section{Acknowledgements}

None.

\section{Conflict of interest}

The author declares no conflict of interest.

\section{References}

1. Nutting S, Carr I, Cole FM, et al. Solitary pulmonary nodules due to sarcoidosis. Can J Surg. 1979;22(6):584-586.

2. Pinsker KL. Solitary pulmonary nodule in sarcoidosis. JAMA. 1978;240(13):1379-1380.

3. Rose RM, Lee RG, Costello P. Solitary nodular sarcoidosis. Clin Radiol. 1985;36(6):589-592.

4. Steele JD. The solitary pulmonary nodule. Report of a cooperative study of resected asymptomatic solitary pulmonary nodules in males. $J$ Thorac Cardiovasc Surg. 1963;46:21-39.

5. Rubinstein I, Baum GL, Lieberman Y, et al. Asymptomatic pulmonary nodule in sarcoidosis. Eur J Respir Dis. 1985;66(1):74-76.

6. Higgins GA, Shields TW, Keehn RJ. The solitary pulmonary nodule. Ten-year follow-up of veterans administration-armed forces cooperative study. Arch Surg. 1975;110(5):570-575.

7. Rockoff SD, Rohatgi PK. Unusual manifestations of thoracic sarcoidosis. AJR Am J Roentgenol. 1985;144(3):513-528. 